\section{FIBROMIALGIA E ATIVIDADE FÍSICA: BENEFÍCIOS E FATORES DE NÃO ADESÃO}

\section{FIBROMYALGIA AND PHYSICAL ACTIVITY: BENEFITS AND FACTORS OF NON-ACCESSION}

\author{
Fábio Fernandes Flores ${ }^{1, *} /$ Berta Leni Costa Cardoso ${ }^{1}$ / \\ Cláudio Bispo de Almeida ${ }^{1} /$ \\ Ricardo Franklin de Freitas Mussi
}

\section{A SÍNDROME DE FIBROMIALGIA E A ATIVIDADE FÍSICA}

A Síndrome de Fibromialgia (SFM) é caracterizada por dores musculoesqueléticas generalizadas crônicas, fadiga, distúrbio do sono, entre outras características, inclusive de caráter psicopatológico (WOLF, 1990 apud CAVALIERE; SOUZA; BARBOSA, 2010, p.1326). Brody e Hall (2012) acrescentam a importância do comprometimento funcional na pessoa acometida, com impacto na deterioração da função respiratória, da amplitude articular e da resistência (endurance) muscular, além da diminuição da força e da aptidão cardiovascular (abaixo dos índices médios).

Ignachewski; Semeghini e Garcia Junior (2004) complementam que o quadro clínico pode ser agravado por: mudanças climáticas e ambientais, flutuações hormonais durante o ciclo pré-menstrual ou menopausa, estresse, depressão, ansiedade e uso exacerbado dos músculos e da atividade cognitiva.

Departamento de Educação - Campus XII, Universidade do Estado da Bahia Guanambi, Bahia - Brasil.

*E-mail para correspondência: fabioedfgbi@gmail.com

\section{RESUMO}

O estudo objetiva analisar os benefícios e fatores de não adesão da prática da atividade física em pessoas com a Síndrome de Fibromialgia. Trata-se de um estudo de revisão integrativa com viés qualitativo e caráter descritivo. Foram realizadas buscas na BVS, Scielo e LILACS, agrupando os trabalhos publicados no período de janeiro de 2010 até março de 2019, utilizou-se os seguintes descritores: Fibromialgia, exercício e atividade física. Dos 252 trabalhos encontrados, 29 foram utilizados, conforme os critérios adotados. Os estudos revisados apontam vários tipos de atividades físicas como benéficas: atividades aeróbicas, aquáticas, rítmicas, de força, flexibilidade e associadas com outras. Nos resultados houve mais benefícios (sono, autonomia, aspecto psicológico, aspecto emocional e bem-estar) do que fatores de não adesão (medo, estudos, evasão e saúde) para a prática da atividade física, tais resultados sugerem a importância da população estudada se tornar fisicamente ativa.

Palavras Chave: Fibromialgia; Atividade física; Benefício. Adesão.

\section{ABSTRACT}

The objective of this study was to analyze the benefits and non-adherence factors of the practice of physical activity in people with Fibromyalgia Syndrome. It is an integrative review study with a qualitative and descriptive bias. We searched the BVS, SciELO and LILACS, grouping the published works from January 2010 to March 2019, using the following descriptors: Fibromyalgia, exercise and physical activity. Of the 252 papers found, 29 were used, according to the criteria adopted. The reviewed studies point to several types of physical activity as beneficial: aerobic, aquatic, rhythmic, strength, flexibility and associated activities. In the results there were more benefits (sleep, autonomy, psychological aspect, emotional aspect and well-being) than factors of non-adherence (fear, studies, avoidance and health) for the practice of physical activity, such results suggest the importance of the study population become physically active.

Keywords: Fibromyalgia; Physical activity; Benefit; Accession.

Submetido em: 23 de jun. 2018

Aceito em: 14 de mai. 2019 
Brody e Hall (2012) informam que é necessário conhecimento aprofundado para a identificação da SFM, decorrente das similaridades de sintomas com doenças reumáticas. Como ainda não existe exame laboratorial, seu diagnóstico baseia-se na identificação de, pelo menos, 11 dos 18 pontos hipersensíveis, sendo eles, nove pontos bilaterais hipersensíveis: occipúcio, cervical, trapézio, supraespinhoso, segunda costela, epicôndilo lateral, glúteo, trocânter maior e joelho. Para um ponto ser considerado hipersensível "positivo" o indivíduo deve referir que a palpação foi dolorosa. Em decorrência da inexistência de cura, o seu tratamento tem como premissa a diminuição do sofrimento, melhora da funcionalidade, autonomia pessoal e qualidade de vida (OLIVEIRA JUNIOR; ALMEIDA, 2018).

O número de pessoas com a SFM tem crescido a cada década. Tal afirmação é advinda da relação de estudos sobre prevalência ao redor do mundo, como mostram Cavalcante et al. (2006), ao apontar que no período de 1990 a 2005 foi identificada uma prevalência na população com valores entre 0,66 e 4,4\%, sendo mais prevalente em mulheres do que em homens. E também Marques et al. (2017), pois indica que entre 2005 a 2014 os valores ficaram entre 0,2 e $6,6 \%$ para a população em geral e, 2,4 e 6,8\% entre mulheres.

Por ser uma manifestação reumática deve ser tratada inicialmente por reumatologistas, sobretudo pelo fato de envolver quadro crônico de dor musculoesquelética. Todavia, o seu tratamento deve ser multidisciplinar, individualizado, ter a participação ativa do paciente, combinar meios farmacológicos e não farmacológicos, como a atividade física (de acordo com a intensidade e características dos sintomas), considerando também as questões biopsicossociais envolvidas no processo (HELFENSTEIN JUNIOR; GOLDENFUM; SIENA, 2012). Flores; Almeida e Mussi (2011) destacam a importância da prática da atividade física (AF), devido à melhoria das capacidades físicas, composição corporal, aspectos relacionados ao social/psíquico/cognitivo, além de interferências positivas nos diversos sistemas do organismo.

A AF regular representa importante fator na melhoria da qualidade de vida das pessoas (NAHAS, 2017). Ferreira; Martinho e Tavares (2014) demonstraram que tal prática adequadamente orientada diminui os sintomas da SFM, aumenta a possibilidade de bem-estar e melhora a Qualidade de Vida.

No entanto, diante dos sintomas da SFM a pessoa costuma sentir-se desencorajada a execução de qualquer tipo de movimento, consequentemente ocorre o estímulo à adoção do comportamento sedentário. Souto et al. (2013) mencionam que pessoas com SFM ficam desestimulados a praticar qualquer tipo de AF para evitar movimentos nos locais dolorosos, pois temem um possível agravamento da sintomatologia.

Apesar de um estudo de revisão de literatura ter apontado a necessidade da melhoria na apresentação das peculiaridades metodológicas do treinamento para pessoas com SFM conforme a modalidade de exercícios (FLORES; ALMEIDA; MUSSI, 2011), outras investigações demonstraram benefícios aos praticantes de exercícios resistidos (REBUTINI, 2013), caminhada (STEFFENS et al., 2011b; STEFFENS et al., 2012), hidroterapia (HECKER et al., 2011), alongamento e caminhada na esteira ergométrica
(MATSUTANI;

ASSUMPÇÃO; MARQUES, 2012).

Destarte, o presente estudo tem a proposta de analisar os benefícios e fatores de não adesão da prática de atividade física para as pessoas com a Síndrome de Fibromialgia, por meio de uma revisão integrativa da literatura, o qual parte da seguinte questão norteadora: Quais são os benefícios e fatores de não adesão da prática de atividades físicas em pessoas com SFM?

\section{MÉTODO}

$\mathrm{O}$ artigo é um estudo de revisão integrativa (SOUZA; SILVA; CARVALHO, 2010), tendo o viés qualitativo (MINAYO, 1996) e de caráter descritivo (GIL, 1999). Na execução do trabalho foram usadas a Biblioteca Virtual em Saúde (BVS) e as bases de dados do Scientific Electronic Library online (Scielo) e da Literatura LatinoAmericana e do Caribe em Ciências da Saúde (LILACS). O cruzamento das palavras-chave teve Descritores em Ciências da Saúde (DeCS) e Termo Livre (TL) e, o indicador booleano foi and; tendo duas combinações: Fibromialgia (DeCS) and exercício (DeCS) e Fibromialgia (DeCS) and atividade física (TL).

Para obtenção dos dados (Tabela 1) considerou-se os seguintes critérios de inclusão: trabalhos (artigos originais, teses, dissertações, relato de caso ou revisões) publicados entre janeiro de 2010 até março de 2019, publicados em língua portuguesa, com resumo e texto completos disponíveis. Já os critérios de exclusão foram os seguintes: trabalhos relacionados com criança e/ou adolescente, estudos que fazem relação com doença (endócrina, cardíaca, respiratória, circulatória ou muscular) ou outra síndrome e uso 
simultâneo de tratamento medicamentoso com o não medicamentoso.

Os trabalhos encontrados tiveram seus temas analisados, aqueles que atenderam aos critérios tiveram o resumo apreciado para confirmar a relação com o propósito deste artigo, em seguida ocorreu à leitura na íntegra de cada trabalho.

Para organizar os dados optouse em colocar as informações na Tabela 2, tendo os seguintes itens: referência e resultados. No que tange aos resultados, categorizou-se em: Benefícios da prática da atividade física e Fatores de não adesão da prática da atividade física e; de acordo com tais indicativos, foram criadas subcategorias.

A avaliação dos dados coletados na pesquisa tem como norteadora a técnica da análise do conteúdo de Triviños (2006), a qual é considerada um meio para estudar as "comunicações", analisando o conteúdo das mensagens com o auxílio de um conjunto de técnicas.

Tal mecanismo divide-se em três etapas, a pré-análise (organização do material constituindo o corpus), a descrição analítica (o corpus é submetido a um estudo aprofundado, orientado por hipóteses) e fundamentação teórica. Utilizando procedimentos como a codificação, a classificação e a categorização na busca de sínteses convergentes e divergentes de ideias, além da interpretação referencial que se apoia nas informações que iniciou na etapa da pré-análise, tentando desvendar o conteúdo latente.

\section{RESULTADOS E DISCUSSÕES}

Diante das combinações das palavras-chave foram encontrados 252 estudos, todavia, somente 81 se enquadravam nos critérios. Em decorrên- cia da repetição dos trabalhos o número reduziu para 29 , conforme pode ser visualizado na Tabela 1 da presente pesquisa.

As informações foram organizadas em duas categorias, sendo elencadas informações sobre os fundamentos para o incentivo da prática da $\mathrm{AF}$, bem como os cuidados e preocupações para que isso ocorra. Cabe apontar que houve também subcategorias, no sentido de aumentar a sistematização dos resultados, conforme pode ser visto no panorama global, bem como suas especificidades a seguir.

\section{Benefícios da prática de atividade física}

Mediante a apreciação dos trabalhos foram elencados aspectos preponderantes, os quais foram organizados em oito subcategorias, são elas: quadro sintomático, Qualidade de Vida (QV), capacidade funcional, sono, autonomia, aspecto psicológico, aspecto emocional e bem-estar.

O quadro sintomático é uma das preocupações primárias das pessoas acometidas pela SFM, neste sentido estudos revelam melhoria na sintomatologia geral (BERSSANETI, 2010; FERREIRA; MARTINHO; TAVARES, 2014), outros direcionam para a redução da dor, sintoma principal da síndrome (ANTUNES et al., 2016; ARAÚJO et al., 2017; BERSSANETI, 2010; CORREIA et al., 2018; HECKER et al., 2011; KÜMPEL et al., 2016; LETIERI et al., 2013; REBUTINI, 2013; STEFFENS et al., 2011c; VALIM et al., 2013;) e, além da redução álgica, há um trabalho que revela também diminuição do número de tender points (MATSUTANI; ASSUMPÇÃO; MARQUES, 2012). De forma complementar e relacionando com exame laboratorial, o trabalho de
Valim et al., (2013) revela que a caminhada aumenta os níveis de ácido 5hidroxiindolacético (5-HIAA) e serotonina (5-HT), isso poderia explicar porque o exercício aeróbico pode melhorar os sintomas (a dor, ansiedade e depressão) de pacientes com SFM mais que o alongamento.

A QV na contemporaneidade tem sido foco em estudos de populações distintas, inclusive, acerca de indivíduos com a SFM. A AF, neste sentido, tem sido um mecanismo positivo para o seu alcance de forma geral (ANTUNES et al., 2016; ARAÚJO et al., 2017; FERREIRA; MARTINHO; TAVARES, 2014; LETIERI et al., 2013; REBUTINI, 2013; STEFFENS et al., 2011c; STEFFENS et al., 2013; TUROZI et al., 2010). Já Berssaneti (2010) cita o aspecto físico e o emocional sob a perspectiva da AF e os seus benefícios para a QV. No trabalho de Hecker et al. (2011) houve um comparativo entre a hidrocinesioterapia e cinesioterapia, onde os dois tiveram melhorias estatísticas no aspecto social; o primeiro, no aspecto emocional e; o segundo, no aspecto físico.

Há indicativos de que com esta prática ocorre uma elevação na capacidade funcional (ARAÚJO et al., 2017; HECKER et al., 2011; KÜMPEL et al., 2016; LETIERI et al., 2013; REBUTINI, 2013), pois ocorre evolução no aspecto físico (HECKER et al., 2011; LORENA et al., 2015) e progresso na aptidão física (BERSSANETI, 2010; BUENO et al., 2012), contribuindo para a realização das atividades básicas diárias (FERREIRA; MARTINHO; TAVARES, 2014). Além disso, Oliveira et al. (2017) afirmam que com o exercício supervisionado (resistência aeróbica, força e flexibilidade de maneira conjunta) houve melhorias na flexibilidade. 
O sono tem sido objeto de estudo de pesquisas tanto de forma geral quanto específica, pacientes com essa síndrome apresentam declínio na qualidade e eficiência do sono, sendo influenciados pela sua latência e duração, presença de dor e despertar noturno (MORO et al., 2014). Por outro lado, a AF tem se revelado como um agente que melhora tal panorama (CAMPOS et al., 2011; KÜMPEL et al., 2016; MATSUTANI; ASSUMPÇÃO; MARQUES, 2012; REBUTINI, 2013; STEFFENS et al., 2011b; STEFFENS et al., 2013; STEFFENS et al., 2014). De acordo com o estudo de Pasqua et al. (2012), foi possível concluir que a caminhada melhora a qualidade subjetiva e reduz os distúrbios do sono.

A autonomia é uma característica importante no tratamento, pois implica em tomar decisões, como por exemplo, fazer AF. Para tanto, existem alguns pontos que a possibilite e que também contribua para seu alcance, tais como: satisfação, pois a atividade deve ser prazerosa e ainda respeitar as preferências do paciente (BUENO et al., 2012); a construção do conhecimento, para a compreensão da relação positiva entre exercício e saúde (CAVALIERE; SOUZA; BARBOSA, 2010); estratégias de intervenção, por serem meios usados para a adesão, como o bom vínculo entre terapeuta/paciente e a prescrição de exercício individualizado por escrito (STEFFENS et al., 2011a) e; ainda, autoeficácia, maneira de lidar com a síndrome (STEFFENS et al., 2011a; STEFFENS et al., 2012).

$\mathrm{O}$ aspecto psicológico destas pessoas, muitas vezes, encontra-se fragilizado e por este motivo estudos são feitos para analisar o efeito da AF nesse contexto. De forma generalizada, Lorena et al. (2015) apontam que o alongamento melhora o aspecto mental, todavia, outras obras descrevem atividades distintas que especificam outros benefícios nesta dimensão, a exemplo de Letieri et al. (2013) que indicam que a hidrocinesioterapia diminui os sintomas da depressão e; Hecker et al. (2011) apontam que a cinesioterapia e hidrocinesioterapia interferem positivamente na saúde mental, melhorando a autoestima e diminuindo a depressão dos pacientes.

A redução da depressão ocorreu também no trabalho de Steffens et al. (2011c), tendo como intervenção os exercícios Qi Gong (exercício oriental que trabalha relaxamento e alongamento), aeróbicos e de força; em Andrade et al. (2016) com a caminhada; e no de Steffens et al. (2013) com a prática conjunta da caminhada com yoga. Já Matsutani; Assumpção e Marques (2012), num estudo comparativo, revelaram que o alongamento é mais eficaz na depressão e o aeróbico na ansiedade.

Pela sintomatologia já descrita, compreende-se que a dimensão emocional é afetada também, todavia, há trabalhos que afirmam que a $\mathrm{AF}$ minimiza tal efeito, tais como: Hecker et al. (2011), que mostraram que com o uso da hidroterapia os pacientes tiveram melhoras estatísticas no aspecto emocional, enquanto que Steffens et al. (2011b) salientou que por meio da caminhada ocorreram melhorias no humor das mulheres com SFM. Não restringindo tal discussão a esta dimensão, o trabalho de revisão de Ferreira, Martinho e Tavares (2014) apontou a redução do estresse como uma forma de gerar bem-estar; e o de Rebutini (2013), que por meio do exercício resistido, constatou melhoria do controle dos fatores de estresse e consequentemente, melhoria no bem-estar dos pacientes.

\section{Fatores de não adesão para a prática da atividade física.}

Apesar de terem sido mencionadas inúmeras implicações positivas quanto ao ato de fazer AF, independentemente da modalidade, é imprescindível salientar quais limitações podem interferir na adesão e/ou continuidade das pessoas em se tornarem fisicamente ativas. Destarte, as informações foram classificadas em seis subcategorias: quadro sintomático, capacidade funcional, medo, estudos científicos, evasão e QV/saúde.

O quadro sintomático é uma das barreiras a serem superadas. Campos et al. (2011) em seu trabalho identificou que o tempo total de sono da amostra foi em torno de 5 horas, o que pode ser considerado insuficiente e não restaurador para esses pacientes, esta baixa qualidade de sono pode ser influenciada pela baixo nível de AF. De forma geral, a sintomatologia implica no início ou continuidade da atividade física, tais como: maior nível de dor em decorrência da capacidade física (LATORRE-ROMÁN et al., 2012); incidência do aumento dos sintomas na fase inicial do programa de exercício, podendo ocasionar um aumento dos sintomas, principalmente dor e fadiga (BUENO et al., 2012); aumento da intensidade dolorosa e da Percepção Subjetiva de Esforço (PSE) durante a realização do Teste de $\mathrm{Ca}$ minhada de 6 minutos (TC6) (HOMANN et al., 2011) e; indivíduos com mais dores podem ter menos tolerância ao esforço (LORENTE; STEFANI; MARTINS, 2014).

A capacidade funcional é consequência da utilização de várias valências físicas. Em pessoas com essa síndrome a capacidade funcional encontra-se em decréscimo (LORENTE; 
STEFANI; MARTINS, 2014). Ao comparar os resultados do TC6 de mulheres com e sem SFM, o primeiro grupo durante o teste teve exacerbação da PSE e dor, o que não ocorreu com o grupo controle; está menor condição cardiorrespiratória compromete a realização das Atividades de Vida Diária (HOMANN et al., 2011). Resultado similar foi encontrado em Breda et al., (2013), ao destacar que as pessoas com SFM também tiveram o pior desempenho, porém isto pode estar relacionado com o estilo de vida, pois houve relação entre o Nível de Atividade Física e o Desempenho Físico. O trabalho de Latorre-Román et al., (2012), ao comparar a aptidão física de mulheres com e sem SFM, identifica uma deteriorização no primeiro grupo, que pode estar relacionada a um maior impacto da síndrome (maior nível de dor). Além disso, a inserção da AF é uma conduta delicada, uma vez que requer o rompimento das barreiras do sedentarismo (BERSSANETI, 2010).

Diante do entorno da SFM, a confiança em fazer algo é substituída pelo receio. Desta maneira, o medo é uma sensação comum (BREDA et al., 2013; FERREIRA; MARTINHO; TAVARES, 2014). Ocorrendo também no fato de fazer qualquer movimento corporal, ou seja, cinesiofobia (BERSSANETI, 2010; LORENTE; STEFANI; MARTINS, 2014).

Mesmo com inúmeros trabalhos/estudos científicos publicados acerca da temática, o conhecimento deve avançar ainda mais. Sobre isso, Ferreira, Martinho e Tavares (2014) indicam a realização de mais estudos que avaliem de modo longitudinal os efeitos do exercício, pois as pesquisas não trazem descrições sobre os métodos utilizados. Além disso, eles apontam a falta de conhecimento dos pacientes como um problema, pois limita o cuidado pessoal da SFM. Dados favoráveis e desfavoráveis devem ser disseminados, como mostra Berssaneti (2010), ao apontar a ineficiência, porque apenas $17 \%$ da amostra referiram melhora com exercícios físicos.

Embora a literatura verse a despeito da importância de ser ativo, a evasão tem sido uma consequência, um exemplo disso é identificado no trabalho de Steffens et al. (2011a), no qual mencionam que um dos motivos para a evasão é a intolerância a exercícios de alta intensidade.

Portanto, em decorrência dos pontos elencados, a pessoa com SFM apresenta tanto a QV quanto a saúde em níveis menores em comparação com aqueles não acometidos por ela. Ratificando o exposto, Latorre-Román et al. (2012) afirmam que a saúde e a QV destas pessoas geralmente são piores, sendo que as mulheres deste estudo estavam com sobrepeso grau II; já no trabalho de Lorente, Stefani e Martins (2014) foi indicado pior QV nos domínios físico e social. De forma aditiva, relacionando um tipo de $\mathrm{AF}$ com a QV, o estudo de Turozi et al. (2010) descreveu que com o uso de Isostretching (ginástica postural) houve piora nos escores do Questionário sobre o Impacto da Fibromialgia na metade da amostra (duas pessoas).

\section{CONSIDERAÇÕES FINAIS}

Os estudos revisados no presente artigo apontam que há mais benefícios do que fatores de não adesão para a prática da AF. Houve uma diversidade de modalidades que indicaram benefícios: caminhada, o método Watsu, Zumba, treinamento resistido, a cinesioterapia, a hidroterapia, pilates, hidrocinesioterapia, alongamento, Qi Gong, prática conjunta de caminhada e yoga, exercício combinado (resistência aeróbica, força e flexibilidade), tai chi e Isostretching.

Os benefícios da prática de $\mathrm{AF}$ em pessoas com SFM, de acordo com os estudos pesquisados, foram autonomia, aspecto psicológico, aspecto emocional e bem-estar. Por outro lado, os resultados da presente pesquisa mostraram fatores de não adesão à prática de AF em pessoas com SFM, foram eles: medo, estudos científicos, evasão e saúde; tal cenário pode contribuir ao comportamento sedentário.

Embora o quadro sintomático, $\mathrm{QV}$, sono e capacidade funcional tenham sido aspectos presentes nas duas categorias, houve muito mais informações relacionadas aos benefícios.

A discussão sobre a relação da AF com a SFM deve continuar, sobretudo, no número de pesquisas com metodologia de intervenção mais descritiva. Este conhecimento com outros coadunam para uma ação consistente no que tange ao trato da pessoa com SFM, e desta forma, torná-la fisicamente ativa. 
Tabela 1. Resultado quantitativo da revisão.

\begin{tabular}{llcc}
\hline \multicolumn{1}{c}{ Fonte } & \multicolumn{1}{c}{ Palavras-chaves } & $\begin{array}{c}\mathbf{N}^{\mathbf{0}} \text { total } \\
\text { de trabalhos }\end{array}$ & $\begin{array}{c}\mathbf{N}^{\mathbf{0}} \text { de trabalhos } \\
\text { que atenderam os critérios }\end{array}$ \\
\hline SCIELO & fibromialgia and atividade física & 14 & 5 \\
SCIELO & fibromialgia and exercício & 14 & 8 \\
LILACS & fibromialgia and atividade física & 45 & 7 \\
LILACS & fibromialgia and exercício & 57 & 22 \\
BVS & fibromialgia and atividade física & 54 & 14 \\
BVS & fibromialgia and exercício & 68 & 25 \\
& $\quad$ TOTAL DE TRABALHOS & & 81 \\
\hline
\end{tabular}

Fonte: Elaborado pelos autores.

Tabela 2. Descrição dos estudos para a revisão que relacionaram SFM e AF publicados no período de janeiro de 2010 até março de 2019.

\begin{tabular}{|c|c|c|}
\hline \multirow[t]{2}{*}{ Referência } & \multicolumn{2}{|l|}{ Resultados } \\
\hline & Benefícios & Fatores de não adesão \\
\hline $\begin{array}{l}\text { Andrade et al } \\
(2016)\end{array}$ & Caminhada na redução nos níveis de depressão & \\
\hline $\begin{array}{l}\text { Antunes et al } \\
(2016)\end{array}$ & $\begin{array}{l}\text { O método Watsu (terapia aquática): } \\
\text { - redução da dor. } \\
\text { - QV }\end{array}$ & \\
\hline Araújo et al (2017) & $\begin{array}{l}\text { Zumba: } \\
\text { - melhora da dor. } \\
\text { - capacidade funcional. } \\
\text { - QV }\end{array}$ & \\
\hline Berssaneti (2010) & $\begin{array}{l}\text { Alongamento e fortalecimento: } \\
\text { - Dor } \\
\text { - sintomas da SFM } \\
\text { - QV (físico e emocional) }\end{array}$ & $\begin{array}{l}\text { - sedentarismo } \\
\text { - cinesiofobia. } \\
\text { - Ineficiência }\end{array}$ \\
\hline Breda et al (2013) & & $\begin{array}{l}\text { - Mulheres com SFM tem pior desempe- } \\
\text { nho físico no TC6 do que as que não têm. }\end{array}$ \\
\hline Bueno et al (2012) & $\begin{array}{l}\text { - Satisfação } \\
\text { - Condicionamento físico }\end{array}$ & Aumento dos sintomas. \\
\hline $\begin{array}{l}\text { Campos et al } \\
(2011)\end{array}$ & O aumento da AF melhora o sono. & O tempo de sono da amostra ( 5 horas) \\
\hline $\begin{array}{l}\text { Cavaliere, Souza e } \\
\text { Barbosa (2010) }\end{array}$ & $\begin{array}{l}\text { Conhecimento (relação entre exercício físico e saúde) } \\
\text { para adesão ao tratamento. }\end{array}$ & \\
\hline Correia et al (2018) & O treinamento resistido reduz a dor. & \\
\hline
\end{tabular}


Ferreira, Martinho

e Tavares (2014)

A prática da $\mathrm{AF}$ regular e orientada:

- Diminuição dos sintomas

- Bem estar

- QV.

Hecker et al (2011) - A cinesioterapia e hidroterapia melhoraram a capacidade funcional, saúde mental e dor.

- A cinesioterapia melhorou os aspectos físicos.

- A hidroterapia melhorou os aspectos emocionais.

- Exercícios aeróbicos (baixa intensidade) e alongamento melhoram a QV.

Homann et al

(2011)

kümpel et al (2016) Pilates:

- Dor

- capacidade funcional

- sono

Latorre-Román et

al (2012)

Letieri et al (2013) Hidrocinesioterapia:

- Capacidade funcional

- sintomas depressivos

- Dor

- QV

Lorena et al (2015) Alongamento:

- Melhora física

- Melhora mental.

Lorente, Stefani e

Martins (2014)

Matsutani, Assumpção e Marques (2012)

Oliveira et al (2017)

Pasqua et al (2012)

- Melhoria na dor, número de tender points, sono e

depressão (alongamento são mais eficazes que os aeróbicos).

- Redução da ansiedade (o aeróbicos parece produzir um efeito mais importante que o alongamento).

Exercício supervisionado (resistência aeróbica, força e flexibilidade) melhorou na flexibilidade.

Caminhada melhorou o sono (Qualidade subjetiva e
- Poucos estudos longitudinais sobre os efeitos do exercício.

- A falta de conhecimento das pessoas com SFM sobre a importância da AF colabora na baixa adesão.

- Pesquisas com descrições insuficientes sobre os métodos utilizados.

- Exacerbação da dor e PSE durante o TC6.

- Pior desempenho na avaliação funcional.

Características das mulheres com SFM:

- Sobrepeso grau II

- aptidão física reduzida

- A saúde e a qualidade de vida são geralmente pior.

- Maior intensidade de dor.

- Medo de movimento

- Pior QV (domínios físico e social).

- Decréscimo na capacidade funcional. redução dos distúrbios). 
Rebutini (2013) Treinamento resistido:

- Sono

- Dores

- Capacidade funcional,

- Bem-estar geral,

- Estresse

- QV.

Steffens et al

(2011a)

Steffens et al (2011b)

Steffens et al (2011c)

- auto-eficácia

- Estratégias de intervenção

A caminhada:

- Sono

- humor

- Dor (atividades aeróbias e de resistência muscular localizada).

- Depressão (QiGong, aeróbios e de força).

- QV (exercícios aeróbios, de força e de flexibilidade).

Steffens et al Caminhada orientada melhorou auto-eficácia (administração dos sintomas).

Steffens et al

Prática conjunta de caminhada e yoga:

(2013)

- Qualidade do sono

- Depressão

- QV

Steffens et al (2014)

Os exercícios físicos (aeróbios, alongamento, fortalecimento muscular, em piscina, respiratório e de tai chi) melhoram a qualidade do sono.

Turozi et al (2010) Isostretching: melhora e/o manutenção da QV nos 4 quesitos QV.

Isostretching: piora da QV na maioria dos escores em 2 pacientes.

Valim et al (2013) A caminhada aumenta os niveis de 5-HIAA e 5-HT (isso poderia explicar porque o exercício aeróbico pode melhorar os sintomas de pacientes com SFM mais que o alongamento).

Fonte: Elaborado pelos autores 


\section{REFERENNCIAS}

ANDRADE, Alexandro et al. A prática de caminhada diminui a depressão em pacientes com síndrome da fibromialgia, ConScientiae Saúde, São Paulo, v. 15, n. 2, p. 217-224, 2016. Disponível em: $<$ http://periodicos.uninove.br/index.ph $\mathrm{p}$ ?journal $=$ saude $\&$ page $=$ article $\& o p=v i$ ew\&path\%5B\%5D=6273\&path\%5B\% 5D=3306 > . Acesso em: 22 mar. 2019.

ANTUNES, Mateus Dias et al. Efeitos do Watsu na qualidade de vida e quadro doloroso de idosas com fibromialgia, ConScientiae Saúde, São Paulo, 15, n. 4, p. 636-641, 2016. Disponível em:

$<$ http://periodicos.uninove.br/index.ph $\mathrm{p}$ ?journal $=$ saude $\&$ page $=$ article $\& o p=v i$ ew\&path\%5B\%5D=6756\&path\%5B \% 5D=3438 >. Acesso em: 22 mar. 2019

ARAÚJO, Joyce Thalita Medeiros de et al. Experiência de mulheres com fibromialgia que praticam zumba. Relato de casos, Revista Dor, São Paulo, v. 18, n. 3, jul./set., p. 266-269, $2017 . \quad$ Disponível em: $<$ http://www.scielo.br/scielo.php?scrip $\mathrm{t}=$ sci_arttext\&pid=S1806-

$00132017000300266 \& \ln \mathrm{g}=\mathrm{en} \& \mathrm{nrm}=\mathrm{is}$ o\&tlng=pt $>$. Acesso em: 22 mar. 2019

BERSSANETI, Ana Assunção. Exercício de alongamento e fortalecimento muscular no tratamento de pacientes com fibromialgia: um ensaio clínico randomizado. Tese (Doutorado em Ciências) - Faculdade de Medicina da Universidade de São Paulo. São Paulo, 92p. 2010.

BREDA, Chris Andreissy et al. Nível de atividade física e desempenho físico no teste de caminhada de 6 minutos em mulheres com fibromialgia. Revista Brasileira de Reumatologia, São Paulo, v. 53, n. 3, p. 276-281, 2013. Disponível em: <http://www.scielo.br/pdf/rbr/v53n3/v 53n3a05.pdf >. Acesso em: $24 \mathrm{fev}$. 2019.

BRODY, Lori Thein; HALL, Carrie M. Exercício Terapêutico na busca da função. Tradução de Giuseppe Taran- to. $2^{\text {a }}$ Ed. Rio de Janeiro: Guanabara Koogan, 2012.

BUENO, Roberta Chiden. Exercício físico e fibromialgia. Cadernos de Terapia Ocupacional. São Carlos, v. 20, n. 2, p. 279-285, 2012. Disponível em:

<http://www.cadernosdeterapiaocupaci onal.ufscar.br/index.php/cadernos/artic le/view/631>. Acesso em: 10 jan. 2019.

CAMPOS, Raquel Munhoz da Silveira et al. Fibromialgia: nível de atividade física e qualidade do sono. Motriz, Rio Claro, v. 17, n. 3, p. 468-476, jul./set., 2011. Disponível em: <http://www.scielo.br/pdf/motriz/v17n 3/10.pdf>. Acesso em: 18 jan. 2019.

CAVAlCANTE, Alane Bento. et al. A prevalência de fibromialgia: uma revisão de literatura. Revista Brasileira de Reumatologia, São Paulo, v. 46, n. 1, p. 40-48, jan./fev., 2006. Disponível em: $<\mathrm{http}: / / \mathrm{www}$. scielo.br/scielo.php?scrip $\mathrm{t}=\mathrm{sci}$ arttext\&pid=S0482-

50042006000100009>. Acesso em: 25 nov. 2018.

CAVALIERE, Maria Lúcia Alves; SOUZA, Jerusa Mônica de Abreu; BARBOSA, José Silvio de Oliveira. Representações da relação entre exercício físico e saúde por pacientes fibromiálgicos. Physis Revista de Saúde Coletiva, Rio de Janeiro, v. 20, n. 4, p. 1325-1339, 2010. Disponível em: <http://www.scielo.br/pdf/physis/v20n 4/a14v20n4.pdf>. Acesso em: 12 jan. 2019.

CORREIA, Lidiane Cristina et al. Efeito do treinamento resistido na redução da dor no tratamento de mulheres com fibromialgia: revisão sistemática. Revista Brasileira de Ciência e Movimento, Brasília, v. 26, n. 2, p. 170-175, 2018. Disponível em: <https://portalrevistas.ucb.br/index.ph $\mathrm{p} / \mathrm{RBCM} /$ article/view/7255/pdf $>$.

Acesso em: 22 mar. 2019.

FERREIRA, Gabriele; MARTINHO, Ulisses Guimarães; TAVARES, Maria da Consolação Gomes C. F. Fibromi- algia e atividade física: reflexão a partir de uma revisão bibliográfica. SALUSVITA, Bauru, v. 33, n. 3, p. 433 446, 2014. Disponível em: $<$ https://secure.usc.br/static/biblioteca/ salusvi-

ta/salusvita_v33_n3_2014_art_10.pdf> . Acesso em: 20 jan. 2019.

FLORES, Fábio Fernandes; ALMEIDA, Cláudio Bispo de; MUSSI, Ricardo Franklin de Freitas. Exercício físico como meio de redução dos sintomas da Síndrome de Fibromialgia. Lecturas: Educación Física y Deportes, Buenos Aires, a. 15, n. 153, feb., 2011. Disponível em: $<$ https://www.efdeportes.com/efd153/r educao-dos-sintomas-da-sindrome-defibromialgia.htm>. Acesso em: 20 jan. 2019.

GIL, Antônio Carlos. Métodos e técnicas de pesquisa social. 5.ed. São Paulo: Atlas, 1999.

HECKER, Celina Dani et al. Análise dos efeitos da cinesioterapia e da hidrocinesioterapia sobre a qualidade de vida de pacientes com fibromialgia um ensaio clínico randomizado Fisioterapia em Movimento, Curitiba, v. 24, n. 1, p. 57-64, jan./mar., 2011. Disponível em: <http://www.scielo.br/pdf/fm/v24n1/v 24n1a07.pdf>. Acesso em: 17 jan. 2019.

HELFENSTEIN JUNIOR, Milton; GOLDENFUM, Marco Aurélio; SIENA, César Augusto Fávaro. Fibromialgia: aspectos clínicos e ocupacionais. Revista da Associação Médica Brasileira, São Paulo, v. 58 , n. 3 , p. 358 365, mai.jun., 2012. Disponível em: <http://www.scielo.br/pdf/ramb/v58n3 /v58n3a18.pdf $>$. Acesso em: 29 jan. 2019.

HOMANN, Diogo et al. Redução da capacidade funcional e exacerbação da dor durante o esforço do teste de caminhada de 6 minutos em mulheres com fibromialgia. Revista Brasileira de Fisioterapia, São Carlos, v. 15, n. 6, p. 474-480, nov./dez., 2011. Disponível em: 
<http://www.scielo.br/pdf/rbfis/v15n6/ v15n6a08.pdf >. Acesso em: 15 fev. 2019.

IGNACHEWSKI, Lucienne Eloise Rocha; SEMEGHINI, Tatiana Adamov; GARCIA JUNIOR, Jair Rodrigues. Aspectos Fisiopatológicos e Neuroendócrinos da Síndrome de Fibromialgia. Saúde em Revista, Piracicaba, v. 6, n. 12, p. 55-58, 2004. Disponível em: $<$ https://pt.slideshare.net/AdrianoPires/ aspectos-fisiopatolgicos-e-

neuroendcrinos-da-sndrome-de-

fibromialgia>. Acesso em: 05 fev. 2019.

KÜMPEL, Cláudia et al. Benefício do Método Pilates em mulheres com fibromialgia ConScientiae Saúde, São Paulo, 15, n. 3, p. 440-447, 2016. Disponível

em:

$<$ http://periodicos.uninove.br/index.ph p? journal $=$ saude $\&$ page $=$ article $\&$ op $=$ vi ew\&path\%5B \%5D=6515\&path\%5B \% $5 \mathrm{D}=3357$. Acesso em: 22 mar. 2019.

LATORRE-ROMÁN, Pedro Ángel et al. Análise das capacidades físicas de mulheres com fibromialgia segundo o nível de gravidade da enfermidade. Revista Brasileira de Medicina do Esporte, São Paulo, v. 18, n. 5, p. 308312, set./out., 2012. Disponível em: <http://www.scielo.br/pdf/rbme/v18n5 /05.pdf>. Acesso em: 23 mar. 2019.

LETIERI, Rubens Vinícius et al. Dor, qualidade de vida, autopercepção de saúde e depressão de pacientes com fibromialgia, tratados com hidrocinesioterapia. Revista Brasileira de Reumatologia, São Paulo, v. 53, n. 6, p. 494-500, 2013. Disponível em: <http://www.scielo.br/pdf/rbr/v53n6/v 53n6a08.pdf>. Acesso em: 23 mar. 2019.

LORENA, Suélem Barros de et al. Efeitos dos exercícios de alongamento muscular no tratamento da fibromialgia: uma revisão sistemática. Revista Brasileira de Reumatologia, São Paulo, v. 55, n. 2, p. 167-173, 2015. Disponível em: <http://www.scielo.br/pdf/rbr/v55n2/0 482-5004-rbr-55-02-0167.pdf>. Acesso em: 25 mar. 2019.

LORENTE, Giovana Davi; STEFANI, Lia Fernanda Bocchi de; MARTINS, Marielza Regina Ismael. Cinesiofobia, adesão ao tratamento, dor e qualidade de vida em indivíduos com síndrome fibromiálgica. Revista Dor, São Paulo, v. 15 , n. 2, p. 121-125, 2014. Disponível

em: <http://www.scielo.br/pdf/rdor/v15n2/ pt_1806-0013-rdor-15-02-0121.pdf >

Acesso em: 20 mar. 2019.

MARQUES, Amélia Marques et al. A prevalência de fibromialgia: atualização da revisão de literatura. Revista Brasileira de Reumatologia, São Paulo, v. 57, n. 4, p.356-363, jul./ago., $2017 . \quad$ Disponível em: $<$ http://www.scielo.br/scielo.php?scrip $\mathrm{t}=$ sci_arttext\&pid=S0482-

50042017000400356\&lng=pt\&nrm=is o\&tlng=pt > . Acesso em: 25 mar. 2019.

MATSUTANI, Luciana Akemi; ASSUMPÇÃO, Ana; MARQUES, Amélia Pasqual. Exercícios de alongamento muscular e aeróbico no tratamento da fibromialgia: estudo piloto Fisioterapia em Movimento, Curitiba, v. 25, n. 2, p. 411-418, abr./jun., 2012. Disponível em: <http://www.scielo.br/pdf/fm/v25n2/v 25n2a19.pdf $>$. Acesso em: $13 \mathrm{fev}$. 2019.

MINAYO, Maria Cecília de Souza. $O$ desafio do conhecimento: pesquisa qualitativa em saúde. 4. ed. São Paulo, 1996.

MORO, Florinda Freire et al. Qualidade do sono em pacientes fibromiálgicos Revista Brasileira em Promoção da Saúde, Fortaleza, v. 27, n.1, p. 7276, jan./mar., 2014 Disponível em: <http://periodicos.unifor.br/RBPS/artic le/view/3162>. Acesso em: 22 mar. 2019.

NAHAS, Markus Vinícius. Atividade física, Saúde e Qualidade de vida: conceitos e sugestões para um estilo de vida ativo. $7^{\mathrm{a}}$ ed. Londrina: Midiograf, 2017.
OLIVEIRA JUNIOR, José Oswaldo de; ALMEIDA, Mauro Brito de. O tratamento atual da fibromialgia. Brazilian Journal of Pain, São Paulo, v. 1, n. 3, p. 255-262, jul./set. 2018. Disponível

em: <http://www.scielo.br/pdf/brjp/v1n3/pt _1806-0013-brjp-01-03-0255.pdf>.

Acesso em: 15 mar. 2019.

OLIVEIRA, Leonardo Hernandes de Souza et al. Efeito do exercício físico supervisionado sobre a flexibilidade de pacientes com fibromialgia, Revista Dor, São Paulo, v. 18, n. 2, abr./jun., p. 145-149, 2017. Disponível em: <http://www.scielo.br/pdf/rdor/v18n2/ pt_1806-0013-rdor-18-02-0145.pdf>.

Acesso em: 22 mar. 2019.

PASQUA, Luana Della et al. Efeito da caminhada no sono de pacientes portadores de síndrome da fibromialgia, ConScientiae Saúde, São Paulo, v. 11, n. 4, p. 580-586, 2012. Disponível em:

<http://periodicos.uninove.br/index.ph p? journal $=$ saude $\&$ page $=$ article $\& o p=v i$ ew\&path $\% 5 \mathrm{~B} \% 5 \mathrm{D}=3622 \&$ path $\% 5 \mathrm{~B} \%$ 5D=2407> . Acesso em: 22 mar. 2019.

REBUTINI, Vanessa Zadorosnei et al. Efeito do treinamento resistido em paciente com fibromialgia: Estudo de caso. Motriz, Rio Claro, v. 19, n. 2, p. 513-522, abr./jun., 2013. Disponível em:

<http://www.scielo.br/pdf/motriz/v19n 2/29.pdf>. Acesso em: 19 fev. 2019.

SOUTO, Deyvison Carlos dos Santos et al. Efeitos da atividade física para mulheres com síndrome da fibromialgia: uma revisão de literatura. Lecturas: Educación Física y Deportes, Buenos Aires, a. 17, n. 178, mar., 2013. Disponível em: <https://www.efdeportes.com/efd178/a tividade-fisica-com-sindrome-dafibromialgia.htm>. Acesso em: 15 abr. 2018.

SOUZA, Marcela Tavares de; SILVA, Michelly Dias da; CARVALHO, Rachel de. Revisão integrativa: o que é e como fazer. Revista Einstein, São Paulo, v. 8, n. 1, p. 102-106, 2010. Disponível 
<http://www.scielo.br/pdf/eins/v8n1/pt _1679-4508-eins-8-1-0102>. Acesso em: 19 nov. 2018.

STEFFENS, Ricardo de Azevedo Klumb et al. Fatores associados à adesão e desistência ao exercício físico de pacientes com fibromialgia: uma revisão. Revista Brasileira de Atividade Física \& Saúde, Pelotas, v. 16, n. 4, p.353-357, 2011a. Disponível em: <http://rbafs.org.br/RBAFS/article/vie w/634/663 >. Acesso em: 17 jun. 2018.

STEFFENS, Ricardo de Azevedo Klumb et al. Praticar caminhada melhora a qualidade do sono e os estados de humor em mulheres com síndrome da fibromialgia. Revista Dor, São Paulo, v. 12, n. 4, p. 327-331, out./dez., 2011b. Disponível em: <http://www.scielo.br/pdf/rdor/v12n4/ a08v12n4.pdf $>$. Acesso em: 15 jun. 2018.

STEFFENS, Ricardo de Azevedo Klumb et al. Exercícios físicos diminuem a dor, a depressão e melhoram a qualidade de vida de pessoas com fibromialgia. ConScientiae Saúde, São Paulo, v. 10, n. 4, p. 749-755, 2011c. Disponível em: $<$ http://periodicos.uninove.br/index.ph p?journal $=$ saude $\&$ page $=$ article $\& o p=v i$ ewFile\&path[]=2770\&path[]=2087>. Acesso em: 17 jun. 2018.

STEFFENS, Ricardo de Azevedo Klumb et al. Efeito da caminhada sobre a qualidade de vida e autoeficácia de mulheres com síndrome da fibromialgia. Revista brasileira de Ciência e Movimento, Brasília, v. 20, n. 1, p. 41-46, 2012. Disponível em: <https://portalrevistas.ucb.br/index.ph p/RBCM/article/view/1894>. Acesso em: 17 jun. 2018.

STEFFENS, Ricardo de Azevedo Klumb et al. Efeito da prática conjunta da caminhada e yoga no sono, depressão e qualidade de vida em pacientes com síndrome da fibromialgia, ConScientiae Saúde, São Paulo, v. 12, n. 3, p. 371-378, 2013. Disponível em: <http://periodicos.uninove.br/index.ph p?journal $=$ saude $\&$ page $=$ article $\&$ op $=v i$ ew\&path\%5B \%5D=4249\&path\%5B\% 5D=2572> . Acesso em: 22 mar. 2019.

STEFFENS, Ricardo de Azevedo Klumb et al. Efeitos dos exercícios físicos no sono de pacientes com síndrome da fibromialgia: revisão sistemática, ConScientiae Saúde, São Paulo, v. 13, n. 2, p. 298-304, 2014. Disponível em: $<$ http://periodicos.uninove.br/index.ph p?journal $=$ saude $\&$ page $=$ article $\&$ op $=$ vi ew\&path\%5B\%5D=4700\&path\%5B\% 5D=2759>. Acesso em: 22 mar. 2019.

TRIVIÑOS, Augusto Nibaldo Silva. Introdução à Pesquisa em Ciências Sociais: A Pesquisa Qualitativa em Educação. São Paulo: Atlas, 2006.

TUROZI, Estela et al. Análise do isostretching na qualidade de vida de pacientes com fibromialgia, Revista Espaço para a Saúde, Londrina, v. 11, n. 2, p. 66-75, jun. 2010. Disponível em: $<$ https://docplayer.com.br/21972532-

Analise-do-isostretching-na-qualidadede-vida-de-pacientes-com-

fibromialgia.html>. Acesso em: 22 mar. 2019.

VALIM, Valéria et al. Efeitos do exercício físico sobre os níveis séricos de serotonina e seu metabólito na fibromialgia: Um estudo piloto randomizado. Revista Brasileira de Reumatologia, São Paulo, v. 53, n. 6, p. 538541, 2013. Disponível em: <http://www.scielo.br/pdf/rbr/v53n6/v 53n6a14.pdf >. Acesso em: 17 jun. 2018. 